\title{
Uso de co-produtos dietéticos no desempenho de coelhos: uma revisão
}

\author{
Use of dietary co-products on rabbit performance: a review \\ Uso de coproductos dietéticos sobre el rendimiento del conejo: una revisión
}

\section{Resumo}

A cunicultura brasileira está em crescente expansão, porém, os custos com alimentação encontram-se elevados. Para garantir a viabilidade econômica no setor cunícola, tem-se desenvolvido pesquisas relacionadas ao uso de alimentos alternativos e co-produtos, visando a diminuição de custos relacionados a dieta. Portanto, objetivou-se através desta revisão de literatura, abordar a utilização de co-produtos e alimentos alternativos na dieta de coelhos, com enfoque no efeito destes no desempenho animal. A revisão foi realizada através de buscas de artigos em bases de pesquisa como PubMed, Science Direct e Google Acadêmico, além de buscas em periódicos específicos da cunicultura. O período de publicação dos artigos estimado para as buscas foi entre 2010 a 2022. A disponibilidade de co-produtos e alimentos alternativos é ampla, porém, para a escolha destes é preciso levar em consideração fatores como a fonte, qualidade e composição química-bromatológica e região. Tais fatores estão relacionados à possíveis efeitos deletérios que podem provocar redução no desempenho dos animais. Após escolhidos, espera-se que a inclusão dietética de tais alimentos promova melhor saúde intestinal dos animais, e consequentemente maior ganho de peso, menor conversão alimentar e influencie no custo de produção da dieta. Conclui-se que há diversidade de ingredientes disponíveis para serem incluídos em dietas para coelhos. No entanto, é necessário realizar estudos contínuos visando determinar com eficiência os níveis de inclusão por fase, bem como a identificação de fatores anti-nutricionais e efeitos negativos presentes em determinados alimentos.

Palavras-chave: Alimentos alternativos; Cunicultura; Ganho de peso; Eficiência produtiva.

\begin{abstract}
The Brazilian rabbit farming is in growing expansion, however, the cost with feeding are high. To ensure economic viability in the rabbit sector, research has been developed related to the use of alternative foods and co-products, aiming to reduce diet related costs. Therefore, the objective of this literature review was to approach the use of coproducts and alternative foods in the diet of rabbits, focusing on their effect on animal performance. The period of publication of articles estimated for the searches was between 2010 and 2022. The availability of co-products and alternative foods is wide, however, when choosing these, factors such as source, quality and chemical-bromatological composition and region must be taken into accout. These factors are related to possible deleterious effects that can lead to reduced animal performance. Once chosen, it is expected that the dietary inclusion of such foods will promote better intestinal health of the animals, and consequently greater weight gain, lower feed conversion and influence the
\end{abstract}


cost of production of the diet. It is conclude that there is a diversity of ingredients available to be included in diets for rabbits. However, it is necessary to carry out continuous studies to efficiently determine the inclusion levels per phase, as well as the identification of anti-nutritional factors and negative effects present in certain foods.

Keywords: Alternative foods; Rabbit farming; Weight gain; Productive efficiency.

\section{Resumen}

La cria de conejos en Brasil se está expandiendo, pero los costos de alimentación son altos. Para asegurar la viabilidad económica de la indústria del conejo, se han realizado investigaciones relacionadas con el uso de alimentos y coproductos alternativos, con el objetivo de reducir los costos relacionados con la dieta. Por tanto, el objetivo de esta revisión de la literatura fue abordar el uso de coproductos y alimentos en la dieta de los conejos, centrándose en su efecto sobre el rendimiento animal. La revisión se realizó mediante búsquedas de artículos en bases de investigación como PubMed, Science Direct y Academic Google, además de búsquedas en revistas específicas de cría de conejos. El período de publicación de los artículos estimado para las búsquedas fue entre 2010 y 2022. La disponibilidad de coproductos y alimentos alternativos es amplia, sin embargo, a la hora de elegi restos se deben tener en cuenta factores como la fuente, la calidad y la composición químico-bromatológica y la región. Tales factores están relacionados con posibles efectos deletérios que pueden reducir el rendimiento de los animales. Una vez elegidos, se espera que la inclusión dietética de dichos alimentos promueva una mejor salud instestinal en los animales, y consecuentemente mayor ganancia de peso, menor conversión alimenticia e influencia en el costo de producción de la dieta. Se concluye que existe diversidad de ingredientes disponibles para ser incluidos en las dietas para conejos. Sin embargo, es necesario realizar estudios continuos para determinar de manera eficiente los niveles de inclusión por fase, así como la identificación de factores antinutricionales y efectos negativos presentes en ciertos alimentos.

Palabras clave: Alimentos alternativos; Cría de conejos; Aumento de peso; Eficiencia productiva.

\section{Introdução}

As dietas representam cerca de $70 \%$ do custo total da produção animal, neste caso, a utilização de alternativas como os co-produtos soma para baratear os custos de produção. Além disso, promovem a utilização de ingredientes que seriam descartados por agroindústrias, quando na verdade poderiam ser incrementados em formulações de rações, garantindo menos poluição ao meio ambiente. Em determinadas regiões do país, a aquisição de ingredientes comumente utilizados em formulações como o milho e soja é dificultada em virtude dos preços, encarecendo o custo. Diante disso, co-produtos como casca de soja, tortas, farelos, bagaços e polpas podem ser incluídos nas formulações, pois possuem capacidade de substituir parcialmente ingredientes tradicionais, além de atender as exigências, contribuindo para o desempenho animal (Viana et al., 2018; Klinger \& Toledo, 2016).

A alimentação de coelhos é baseada em ingredientes constituídos em sua maioria por celuloses, as quais são decompostas por microorganismos do ceco através de eficiente processo de fermentação. A variabilidade de microorganismos são responsáveis pelo sucesso na fermentação, entretanto, devem estar em constante simbiose, garantindo sucesso no processo e saúde intestinal adequada aos animais (Wang et al., 2018). Estudos relacionados à melhorias em dietas na cunicultura tem sido realizados por diversos autores, avaliando a importância de ingredientes como vitaminas e minerais (Abdel-Wareth et al., 2019; Knob et al., 2019) óleos essenciais (Turchi et al., 2019; Mannelli et al., 2018), probiótico e prebióticos (Coelho et al., 2014; Bovera et al., 2012) e aditivos (El-Aziz et al., 2019).

Alimentos que podem ser utilizados como fontes alternativas fibrosas e energéticas como raspa de mandioca, feno de maniçoba, feno de leucena, casca de soja, são estudados por diversos autores. Entretanto, a inclusão dietética destes requer procedimentos para redução de efeitos anti-nutricionais que podem comprometer o aproveitamento da dieta pelos animais. Ademais, o uso da fenação, desidratação e lavagem de algumas fontes fibrosas podem reduzir efeitos negativos quando realizado a inclusão total ou parcial na dieta dos animais (Viana et al., 2018).

Atualmente, busca-se aprimorar o conhecimento sobre a composição química-bromatológica dos ingredientes, e com isso determinar os níveis corretos para inclusão nas dietas. O entendimento do valor nutritivo é fundamental para formulação de dietas, pois torna-se possível formular dietas visando manter exigências nutricionais. Anteriormente, as dietas formuladas 
para os coelhos eram baseadas no conceito de fibra bruta, sendo necessário balancear os níveis de proteína bruta e energia, pois quando há um desbalanço na relação proteína-energia, ocorre desequilíbrio a nível intestinal, impactando no desempenho dos animais. Sendo assim, objetivou-se com o presente estudo reunir informações referentes à utilização de co-produtos em dietas para coelhos.

\section{Metodologia}

Para realização da revisão de literatura do presente estudo foi utilizada a abordagem exploratória, com pressupostos da pesquisa bibliográfica e documental, segundo a metodologia proposta por Pereira et al., (2018), tendo como produto uma revisão de literatura, compilando informações científicas relacionadas à temática do uso de co-produtos dietéticos sobre o desempenho de coelhos. Fez-se seleção de artigos utilizando as seguintes bases: PubMed, Science Direct e Google Acadêmico. Utilizando para busca as palavras-chaves: Alimentos alternativos, Cunicultura, Ganho de peso, Eficiência produtiva. Posteriormente, realizou-se seleção de artigos, onde o período utilizado para escolha das pesquisas foi o mais recente possível (2010 - 2022). Trabalhos utilizados que apresentem ano de publicação inferior ao mínimo estipulado são justificados em função da contribuição dos mesmos para a escrita do artigo.

\section{Desenvolvimento}

Normalmente, as fontes de energia e proteína nas dietas são atendidas com a utilização de ingredientes como milho e soja. Visando a substituição do farelo de soja na dieta, Coloni et al. (2012), testaram a inclusão parcial de farelo de girassol (FG) em rações para coelhos em crescimento sobre o desempenho dos animais. Ao término do estudo, os autores observaram melhora na conversão alimentar em animais que consumiram a dieta experimental contendo FG, seguido de maior consumo de ração. Para os autores, o farelo de girassol pode substituir eficientemente o farelo de soja em até $40 \%$ da dieta total, visto que os animais apresentam peso final similares quando comparados à coelhos alimentados com dieta a base de farelo de soja.

A casca de soja é um dos ingredientes utilizados como co-produto, porém apresenta alta concentração de polissacarídeos não amiláceos (pectinas, hemicelulose e celuloses, mas baixo teor de ligninas). Estes por sua vez necessitam ser degradados de maneira eficiente no intestino grosso, onde a fermentação ocorre com a ação da microbiota. Outro ingrediente que pode ser utilizado na dieta de coelhos em crescimento é a casca de mandioca, podendo ser incluída na dieta em até $24 \%$ (Klinger et al., 2016).

Em estudo, Oliveira et al. (2013), tiveram como objetivo avaliar o uso de dietas simplificadas à base de feno de alfafa e do terço superior da rama da mandioca. Neste estudo, observou-se que coelhos da raça Nova Zelândia não apresentaram efeitos significativos nos coeficientes de digestibilidade ao comparar com a dieta referência do estudo, onde digestibilidade aparente de matéria seca, proteína bruta e energia bruta foram menores. Ademais, índices de desempenho como ganho de peso e conversão alimentar, foram melhores em animais que receberam a dieta referência. Por fim, os autores relataram que a presença de fatores anti-nutricionais pode ter influenciado nos resultados obtidos.

A alimentação de coelhos baseia-se na utilização de ingredientes como forragens e fontes alternativas de fibra, como é o caso do feno de alfafa, que não afeta o desempenho dos animais. A fibra dietética tem sua composição por polissacarídeos estruturais (celulose e hemicelulose), sendo associadas à substâncias pécticas e compostos fenólicos. Além disso, a inclusão de fibra na dieta de coelhos favorece a manutenção da microbiota dos animais, o funcionamento como substrato garante uma fermentação microbiana, influenciando positivamente no ecossistema simbiótico dos animais (Delgado et al., 2019).

Em estudo, Coloni et al. (2012), buscaram utilizar a substituição parcial e total do feno de alfafa pela polpa cítrica em rações para coelhos em crescimento. Os autores observaram queda na digestibilidade da proteína bruta quando adicionado a 
polpa cítrica nas dietas, os mesmos justificaram que a composição da polpa provoca aumento das substâncias pécticas e diminuição do grau de lignificação das dietas, associado a uma excreção de cecotrofos pelos animais. Quanto ao desempenho, observou-se que, dietas contendo $75 \%$ da polpa cítrica, promoveram maior ganho de peso. Por outro lado, dietas que não continham polpa cítrica, proporcionaram maior consumo de ração, estando este resultado associado ao alto teor de fibra presente no feno de alfafa.

Plantas como a Moringa oleifera e Leucaena leucocephala são utilizadas na nutrição animal como ingredientes alternativos, principalmente por causa do alto teor de proteína (20-25\%), constituído por conteúdo elevado de aminoácidos essenciais. Em dietas para coelhos em crescimento, não se observou efeitos significativos para parâmetros como ganho de peso e conversão alimentar. Entretanto, a inclusão de 30\% pode baratear os custos de produção de alimentação (Santos et al., 2017).

Safwat et al. (2014), estudaram os efeitos suplementação com Leucaena leucocephala (LLM) ou folhas de Moringa oleiferafarinha (MO), com fontes alternativas de alimentos (30 ou 40\%), na dieta de coelhos em crescimento. Neste estudo, avaliou-se os efeitos da suplementação sobre desempenho de crescimento, característica de carcaça e eficiência econômica de coelhos em crescimento. Ao final do período experimental, evidenciou-se que animais tratados com MO apresentaram maior ganho médio diário e maior peso corporal final. Além disso, os autores relatam que a suplementação com MO proporcionou melhor taxa de conversão alimentar na inclusão de $30 \%$ do alimento.

Ingredientes como cevada, trigo, milho ou sorgo, também são considerados alimentos alternativos oriundos da agroindústria. Estes podem ser incluídos em dietas para coelhos visando a redução do custo. Em estudo, Yang et al. (2018), relataram que a utilização do sorgo oriundo do processo de destilaria de grãos em substituição ao milho na dieta de coelhos, não apresentou efeitos significativos sobre o desempenho animal. Além disso, houve redução linear no peso e rendimento de carcaça em animais que consumiram níveis crescentes de sorgo oriundos da destilaria. Este estudo sugere que a incorporação de enzimas exógenas via dieta poderia promover aumento no aproveitamento da ração.

Os óleos essenciais, polpas e determinadas frutas, também estão presentes na nutrição de coelhos. Um exemplo é adição de romã (Punica granatum), que possui ação modulatória antioxidante e imunológica, melhorando a saúde dos animais, pois atua como antioxidante natural em função da presença de compostos polifenólicos na casca e sementes do fruto (Derakhshan et al., 2018). Hassan et al. (2020), utilizaram em seu estudo o extrato de subprodutos de romã em diferentes níveis de inclusão na dieta (100-, 150 e 200 mg / kg), e observaram que o melhora no peso corporal final dos animais com adição do co-produto. Entretanto, efeitos significativos na carcaça dos animais em ambos os tratamentos não foram observados. Por outro lado, houve redução na incidência de E. coli e salmonella no ceco dos animais avaliados, quando submetidos a dietas contendo inclusão da romã, demonstrando o efeito antimicrobiano que a mesma possui.

Com a diminuição do uso de antibiótico na dieta dos animais, ingredientes fitogênicos tornaram-se importante alternativas para reduzir desafios diários, pois contribuem para melhora no desempenho e saúde dos animais através do conceito de nutrição funcional. A inclusão de pimentas na dieta de animais monogástricos contribui apresentam ação antioxidante, antimicrobiana, atividades antifúngicas e anti-inflamatórias, melhorando a absorção e digestão, e a termogênese lipídica (El-Hack et al., 2019).

El-Hack et al. (2019), avaliaram o efeito da inclusão de mistura de óleos de pimentas vermelhas (Capsulam Annum L.) e preta na dieta de coelhos desmamados. Ao término da pesquisa, constatou-se efeito positivo para aumento no peso nas primeiras semanas em animais alimentados com maior nível de inclusão do ingrediente ( $1.5 \mathrm{~g} / \mathrm{kg}$ dieta). Por outro lado, coelhos alimentados com tratamento controle (somente pimenta preta), não apresentaram aumento no ganho de peso. O aumento no ganho de peso é explicado em função da presença do composto ativo Capsaicina, o qual apresenta rica fonte de 
vitamina $\mathrm{C}$, que proporciona aumento na ingestão de alimento, refletindo na melhora do peso corporal. Entretanto, quando avaliado a carcaça dos animais, não se observou resultados significativos.

Ramas de batata-doce foram utilizadas em estudo realizado por Klinger et al. (2020), em substituição ao feno de alfafa para coelhos a partir dos 35 dias de vida. Neste estudo, comprovou-se que dietas contendo ramas de batata doce possuíam maior tempo de retenção, contribuindo para aumento na disponibilidade de nutrientes. Além disso, apresentavam em sua composição melhor fibra para degradação, em virtude do baixo teor de lignificação. Ademais, a substituição de até $15 \%$ do feno de alfafa na dieta não interfere no desempenho dos animais.

A digestibilidade dos ingredientes que compõe a dieta também é avaliada, visando elucidar o aproveitamento de nutrientes. Ferreira et al. (2021), avaliando o uso da semente de maracujá na dieta de coelhos, observaram aumento da digestibilidade da proteína bruta e do extrato etéreo. Neste mesmo estudo, o ganho de peso corporal, peso final e peso da carcaça foram melhores em animais que consumiram dieta contendo semente de maracujá. Além disso, este produto mostrou ser eficiente composto antioxidante na carne de coelho, concluindo que além destes resultados, o custo da dieta é reduzido ao se utilizar a inclusão do coproduto.

Em busca de substituir o farelo de soja (FS) na dieta de coelhos, Garcia Santos et al. (2021), utilizaram as sementes de lupino inteira (Lupinose albas e Lupinus luteus) como uma fonte alternativa de proteína bruta. Entretanto, observaram maior ganho de peso e taxa de conversão de ração maior na fase total de experimento quando analisado o tratamento contendo FS. Contudo, os autores concluíram que a substituição do ingrediente na dieta dos animais não apresenta impactos negativos no desempenho, ainda que mais estudos sejam necessários com a utilização de sementes de lupino na dieta de coelhos.

Chen et al. (2019), buscando utilizar folhas de ervilha em substituição ao feno de alfafa em dieta para coelhos, visto que o valor nutricional das folhas é similar entre os dois alimentos, observaram que não houve diferença na ingestão diária de alimento e ganho médio diário de peso. A inclusão da folha de ervilha influenciou na melhora da taxa de conversão de alimentar, além de ser considerada fonte promissora de proteína.

Strychalski et al. (2014), utilizando resíduos da indústria (resíduos de colza e grãos de destilarias secos de trigo), não observaram resultados significativos no desempenho dos animais nos diferentes tratamentos de inclusão dos co-produtos. Quando avaliado a digestibilidade de nutrientes, constatou-se melhora na digestibilidade de matéria seca e orgânica, proteína bruta, extrato de etéreo e energia nos animais do grupo controle. Os autores citam a necessidade de verificar os níveis de micotoxinas nos co-produtos. Este estudo comprova a necessidade de conhecimento do ingrediente a ser utilizado na formulação, pois podem promover ou não melhoras na digestibilidade e desempenho produtivo dos animais.

No Brasil, a diversidade de plantas tropicais favorece a inclusão destas nas dietas para animais de produção. Algumas plantas possuem alto rendimento de biomassa e composição química bromatológica ideal que favorecem a inclusão (Bacab et al., 2015). O uso de beldroegas (Portulaca oleracea) na dieta de coelhos foi estudada, visando avaliar os efeitos da mesma sobre o desempenho dos animais. Foram utilizados tratamentos contendo ou não a planta. Ao término da pesquisa, conclui-se que não houve efeito positivo no ganho de peso, conversão alimentar e rendimento de carcaça. Entretanto, os autores sugeriram que a planta pode ser incluída em níveis em torno de 30\% em dietas para coelhos (Bacab et al., 2015).

Dabbou et al. (2019), utilizando subproduto do processamento de frutas industrial (bagaço de frutas), na dieta de coelhos não observaram efeitos significativos para ganho de peso e ingestão de ração no período experimental. Porém, quando se considerou a ingestão diária de ração e conversão alimentar durante todo período experimental, constatou-se redução no fornecimento de ração. Os autores citam que o bagaço de frutas utilizado apresentava excelente nível de proteína bruta, além de atuar como fonte de fibra, justificando neste caso o baixo nível de inclusão na dieta para diminuir os efeitos anti-nutricionais de taninos ou outros componentes. A redução na conversão alimentar pode ser explicada em função da presença de ácidos orgânicos (málico, cítrico, entre outros), os quais aumentam a palatabilidade da dieta. 
Gomes et al. (2021), ao verificar a influência da inclusão de níveis crescentes da casca de maracujá (0, 8, 16, 24 e $32 \%$ ) sobre o desempenho de coelhos na fase de crescimento verificou que não a substituição da ração comercial pelas doses de casca de maracujá não alterou o ganho de peso dos animais no final do período experimental, entretanto o grupo que recebeu 32\% da casca de maracujá na dieta apresentou um menor consumo de matéria seca que é explicado devido aos altos níveis de FDN encontrado no material que resulta em um menor consumo de matéria seca matéria, principalmente devido à sensação de saciedade, e através da redução do esvaziamento gástrico e trânsito intestinal. Demonstrando que a inclusão de $32 \%$ desse coproduto apresenta potencial como substituto de ingredientes tradicionais na formulação de dieta de coelhos na fase de crescimento reduzindo o custo com alimentação sem prejudicar o desempenho e as características de carcaça dos animais.

O uso de aditivos medicinais para rações é utilizado com o intuito de aliviar o estresse oxidativo induzido pelo estresse térmico em coelhos. Baseando-se deste pressuposto, Khairy Amber et al. (2021), avaliou o possível papel protetor da raiz de gengibre (Zingiber officinale) contra o estresse calórico em coelhos, para isso foram utilizados animais com cinco semanas de idades alimentados com dieta de engorda suplementada com 0, 2,5, 5,0 e 7,5 g de pó de gengibre / kg de dieta. A temperatura e a umidade relativa durante o período experimental foram mantidas em $33,0 \pm 5,5{ }^{\circ} \mathrm{C}$ e $74,5 \pm 4,5 \%$, respectivamente, durante 8 semanas.

Os autores verificaram que os animais que receberam a dieta suplementada com $7,5 \mathrm{~g}$ de raiz de gengibre apresentou maior ganho de peso ao final do período, foi observado também que os grupos tratados com a raiz do gengibre apresentaram menor taxa de mortalidade durante o período quando comparados ao grupo controle, sendo que as doses de 5,0 e 7,5 g do pó do gengibre resultaram em seus devidos grupos uma maior capacidade antioxidante total do plasma e uma menor concentração plasmática de malondialdeído quando comprado aos alimentados com a dieta controle. Com base nos resultados obtidos conclui-se que a suplementação de gengibre em pó até 5,0g/kg da dieta resultado em uma melhoria do desempenho e aumenta a viabilidade de coelhos expostos a condições de estresse por calor.

Garcia et al. (2021), avaliou o desempenho produtivo e a viabilidade econômica da utilização de silagem de milho e silagem de girassol na alimentação de coelhos em fase de crescimento. Ao final dos 100 dias do período experimental os autores observaram que os animais que receberam o tratamento com a silagem reduziram o consumo de água em cerca de $20 \%$ quando comprado ao grupo controle, este fato é explicado devido a composição bromatológica das dietas, uma vez que as dietas contendo silagem apresentaram uma umidade que variava de 71 a $66 \%$, enquanto a dieta controle possuía apenas $12 \%$ de umidade.

$\mathrm{Na}$ avaliação do desempenho animal não foi observado diferenças entre o ganho de peso diário dos grupos avaliados, entretanto os animais alimentados com silagem de milho apresentaram uma redução de $20 \%$ no consumo de matéria seca quando comparado ao grupo controle, fator esse que acarretou uma redução no custo com alimentação dos coelhos alimentados com a dieta contendo silagem de milho.

Em estudo realizado por Dal Bosco et al. (2012), avaliou-se os efeitos da adição via dieta de três tipos diferentes de bagaço de azeitona desidratado e sem caroço sobre o desempenho produtivo e qualidade da carne de coelhos em crescimento. Neste estudo, constatou-se que a adição de 5\% do bagaço de azeitona na dieta dos coelhos melhorou significativamente o valor nutricional da carne. Além disso, observou-se que este tipo de alimento enriquece a qualidade com a presença de ácidos graxos monoinsaturados (ácido oleico), que apresentam beneficios para a saúde humana.

Kovitvadhi et al. (2016), estudaram os efeitos da suplementação dietética com Echinacea pallida (EPAL), uma planta herbácea, na dieta de coelhos em crescimento sobre os parâmetros de desempenho de crescimento, comunidade bacteriana, sanguíneos e imunidade. Ao término da pesquisa, os autores evidenciaram que a suplementação não provocou efeitos sobre os parâmetros avaliados. Entretanto, foi observado aumento na atividade de fagocitose. 
Como co-produtos, também há possibilidade de se utilizar a torta de macaúba, alimento esse bastante difundido em pesquisas (Ferreira et al., 2018), havendo estudos na área da bovinocultura e caprinocultura (Azevedo et al., 2013), e suinocultura (Costa Júnior et al., 2015). O resíduo restante da extração do óleo da macaúba é um coproduto de fácil encontro nas regiões do semiárido brasileiro. Tal produto por se tratar de um resíduo pode ser usado na dieta animal com a finalidade de se diminuir os custos, substituindo assim o milho nas mesmas (Azevedo et al., 2014).

Há uma certa escarces de pesquisas que envolvam a Macaúba na alimentação de coelhos, no entanto autores desenvolveram pesquisas utilizando bolo de semente da Macaúba na alimentação de desses animais em quatro tratamentos, da qual se dividiram em 0, 50, 100 e 150 gramas desse coproduto na dieta. Tal pesquisa evidencia que a inclusão desse coproduto reduz linearmente a conversão alimentar e o consumo diário de ração, além de manter por igual entre os tratamentos testados a mesma média de ganho de peso diário (Ferreira et al., 2018).

A utilização de alternativas alimentares, visando melhorias em parâmetros de desempenho e qualidade do esterco em coelhos no período pós desmame foi estudada. Na oportunidade, avaliou-se os efeitos da suplementação de folhas de Nim indiano desidratadas na dieta de coelhos após o período de desmame sobre o ganho de peso médio diário (GMD), e qualidade do esterco. Após o término da pesquisa, constatou-se que a suplementação não causou efeitos significativos no GMD, porém, a análise realizada sobre o esterco revelou rica presença de minerais (Fabiano \& Serafim, 2015).

\section{Conclusão}

O uso de co-produtos na dieta de coelhos pode auxiliar como alternativa para diminuir os custos em dietas, sendo necessário verificar a composição nutricional dos ingredientes, visando atender à exigência dos animais, não provocando efeitos negativos no desempenho dos animais. O Brasil apresenta variedade de ingredientes que podem ser introduzidos na dieta de coelhos, porém, novos estudos devem ser realizados visando elucidar os efeitos de cada um deles, garantindo níveis ideais na inclusão dos mesmos em dietas. Adicionalmente, fatores anti-nutricionais e efeitos negativos decorrentes destes precisam ser melhor detalhados.

\section{Referências}

Abdel-Wareth, A. A. A., Ahmed, A. E., Hassan, H. A., Abd El-Sadek, M. S., Ghazalah, A. A., \& Lohakare, J. (2019). Nutritional impact of nano-selenium, garlic oil, and their combination on growth and reproductive performance of male Californian rabbits. Animal Feed Science and Technology, 249(3), 37-45. https://doi.org/10.1016/j.anifeedsci.2019.01.016.

Amber, K., Badawy, N. A., El-Sayd, A. E-N. A., Morsy, W. A., Hassan, A. M., \& Dawood, M. A. O. (2021). Ginger root powder enhanced the growth productivity, digestibility, and antioxidative capacity to cope with the impacts of heat stress in rabbits. Journal of Thermal Biology, 100(8), 1-7. https://doi.org/10.1016/j.jtherbio.2021.103075.

Azevedo, R. A., Santos, A. C. R., Ribeiro Júnior, C. S., Santos, F. P. C., Araújo, L., Bicalho, F. L., Fonseca, L. M., \& Geraseev, L. C. (2014). Performance of dairy cows fed diets containing macauba meal. Arquivos Brasileiro de Medicina Veterinária e Zootecnia, 66(1), 211-218. 10.1590/S010209352014000100029.

Bacab, L. E. C., Franco, L. S., \& Castro, C. S. (2015). Efeito da inclusão dietética de beldroegas no desempenho e conteúdo de ácidos graxos da carne de coelhos em crescimento. Revista Brasileira de Cunicultura, 8(1), 1-12. https://doi.org/10.2527/jas.2011-4119.

Bovera, F., Lestingi, A., Iannaccone, F., Tateo, A., \& Nizza, A. (2012). Use of dietary mannanoligosaccharides during rabbit fattening period: Effects on growth performance, feed nutrient digestibility, carcass traits, and meat quality. Journal of Animal Science, 90(11), 3858-3866. https://doi.org/10.2527/jas.2011-4119.

Chen, Y., Zhu, N., Chen, X., Liu, G., Li, Y., Guo, Y., Deng, M., Liu, D., \& Sun, B. (2019). Evaluation of pigeon pea leaves (Cajanus cajan) replacing alfalfa meal on growth performance, carcass trait, nutriente digestibility, antioxidant capacity and biochemical parameters of rabbits. Journal of Animal Physiology and Animal Nutrition, 103(9), 1265-1273. https://doi.org/10.1111/jpn.13119.

Coelho, C. C. G. M., Mota, K. C. N., Ferreira, F. N. A., Neta, C. E. S., Machado, L. C., \& Ferreira, W. M. (2014). Aditivos equilibradores de flora intestinal para coelhos. Revista Brasileira de Cunicultura, 5(1), 1-20.

Coloni, R. D., Lui, J. F., Sugohara, A., Ezequiel, J. M. B., Morelli, M., \& Bedore, L. (2012). Farelo de girassol em substituição ao farelo de soja em rações de coelhos em crescimento. Revista Brasileira de Cunicultura, 2(1), 1-14. 
Coloni, R. D., Lui, J. F., Sugohara, A., Ezequiel, J. M. B., Morelli, M., \& Bedore, L. (2012). Polpa cítrica em substituição ao feno de alfafa em rações de coelhos em crescimento. Revista Brasileira de Cunicultura, 2(1), 1-13, 2012.

Dabbou, S., Ferrocino, I., Kovitvadhi, A., Dabbou, S., Bergagna, S., Dezzuto, D., Schiavone, A., Cocolin, L., Gai, F., Santoro, V., \& Gasco, L. (2019). Bilberry pomace in rabbit nutrition: effects on growth performance, apparent digestibility, caecal traits, bacterial community and antioxidant status. Animal, 13(1), 53-63. https://doi.org/10.1017/S175173111800099X.

Dal Bosco, A., Mouvarki, E., Cardinali, R., Servili, M., Sebastiani, B., Ruggeri, S., Mattioli, S., Taticchi, A., Esposto, S., \& Castellini, C. (2012). Effect of dietary supplementation with olive pomaces on the performance and meat quality of growing rabbits. Meat Science, 92(4), 783-788. https://doi.org/10.1016/j.meatsci.2012.07.001.

Delgado, R., Menoyo, D., Abad-Guamán, R., Nicodemus, N., Carabaño, R., \& García, J. (2019). Effect of dietary soluble fibre level and n-6/n-3 fatty acid ratio on digestion and health in growing rabbits. Animal Feed Science Technology, 255(8), 1-11. https://doi.org/10.1016/j.anifeedsci.2019.114222.

Derakhshan, Z., Ferrante, M., Tadi, M., Ansari, F., Heydari, A., Hosseini, M. S., Conti, G. O., \& Sadrabad, E. K. (2018). Antioxidant activity and total phenolic content of ethanolic extract of pomegranate peels, juice and seeds. Food and Chemical Toxicology, 114(4), 108-111. https://doi.org/10.1016/j.fct.2018.02.023.

El Azziz, A. H. A., El-Kasrawy, N. I., Ghanima, M. M. A., Alsenosy, A. E-W. A. E., Raza, S. H. A., Khan, S., Memon, S., Khan, R., \& Ullah, I. (2020). Influence of multi-enzyme preparation supplemented with sodium butyrate on growth performance blood profiles and economic benefit of growing rabbits. Journal of Animal Physiology and Animal Nutrition, 104(1), 186-195. https://doi.org/10.1111/jpn.13227.

El-Hack, M. E. A., Alagawany, M., \& Abdelnour, S. (2019). Responses of growing rabbits to supplementing diet with a mixture of black and red pepper oils as a natural growth promoter. Journal of Animal Physiology and Animal Nutrition, 103(2), 509-517. https://doi.org/10.1111/jpn.13045.

Fathi, M., Abdelsalam, M., Al-Homidan, I., Ebeid, T., El-Zarei, M., \& Abou-Emera, O. (2017). Effect of probiotic supplementation and genotype on growth performance, carcass traits, hematological parameters and immunity of growing rabbits under hot environmental conditions. Animal Science Journal, 88(10), 1644-1650. https://doi.org/10.1111/asj.12811.

Ferreira, A. C. S. Watanabe, P. H., Mendonça, I. B., Ferreira, J. L., Nogueira, B. D., Vieira, A. V., Pinheiro, R. R. S., Barros, T. C. R. S., Zampieri, L. A., Vieira, E. H. M., Gomes, T. R., Batista, A. S. M., Leite, S. C. B., \& Freitas, E. R. (2021). Effects of passion fruit seed (Passiflora edulis) on performance, carcass traits, antioxidant activity, and meat quality of growing rabbits. Animal Feed Science and Technology, 275(5), 1-13. https://doi.org/10.1016/j.anifeedsci.2021.114888.

Ferreira, W. M. Ferreira, F. N. A., Inácio, D. F. S., Mota, K. C. N., Júnior, M. B. C., Neta, C. S. S., Rocha, L. F., \& Miranda, E. R. (2018). Effects of dietary inclusion of macaúba seed cake meal on performance, caecotrophy traits and in vitro evaluations for growing rabbits. Archives Animal of Nutrition, 72(2), 138152. https://doi.org/10.1080/1745039X.2018.1435480.

Garcia, R. P. A., Vieira, M. M., \& Soares, D. B. (2021). Desempenho produtivo e análise econômica de coelhos em crescimento suplementados com silagem de milho ou silagem de girassol. Pesquisa Agropecuária Gaúcha, 27(1), 99-109. https://doi.org/10.36812/pag.202127199-109.

Gomes, T. R. Freitas, E. R., Watanabe, P. H., Ferreira, A. C. S., \& Tavares, L. M. S. (2021). Casca do maracujá na alimentação de coelhos em crescimento. Revista Ciência Agronômica, 52(4), 1-11. https://doi.org/10.5935/1806-6690.20210051.

Hassan, F. A., Ibrahim, M. R. M., \& Arafa, S. A. (2020). Effect of dietary pomegranate by-product extract supplementation on growth performance, digestibility, and antioxidant status of growing rabbit. Tropical Animal Health and Production, 52(7), 1893-1901. https://doi.org/10.1007/s11250-020-022010 .

Klinger, A. C. K. Falcone, D. B., Prates, L. S. A., Toledo, G. S. P., \& Silva, L. P. (2020). Digestibility of sweet potato vines in diets for growing rabbits. Revista Brasileira de Cunicultura, 18(1), 1-10. 10.46342/cunicultura.v1.2020-6.

Klinger, A. C. K., \& Toledo, G. S. P. (2016). Ingredientes não convencionais na nutrição cunícula no Brasil - Uma revisão. Revista Brasileira de Cunicultura, $9(1), 1-7$.

Knob, A. N., Klinger, A. C. K., Bortoluzzi, D. F., \& Toledo, G. S. P. (2019). Importância dos minerais na nutrição de coelhos. Revista Brasileira de Cunicultura, 15(1), 1-17.

Kovitvadhi, A., Gai, F., Dabbou, S., Ferrocino, I., Rotolo, L., Falzone, M., Vignolini, C., Gennero, M. S., Bergagna, S., Dezzutto, D., Barbero, R., Nebbia, P., Rosati, S., Cocolin, L., Zoccarato, I., \& Gasco, L. (2016). Rabbit dietary supplementation with pale purple coneflower. 2. Effects on the performances, bacterial community, blood parameters and immunity of growing rabbits. Animal, 10(7), 1110-1117. 10.1017/S1751731115002980.

Mannelli, F., Cappucci, A., Pini, F., Pastorelli, R., Decorosi, F., Giovannetti, L., Mele, M., Minieri, S., Conte, G., Pauselli, M., Rapaccini, S., Viti, C., \& Buccioni, A. (2018). Effect of different types of olive oil pomace dietary supplementation on the rumen microbial community profile in Comisana ewes. Scientific Reports, 8, 1-11. https://doi.org/10.1038/s41598-018-26713-w.

Oliveira, C. E. A., Ferreira, W. M., Ferreira, F. N. A., Hosken, F. M. (2013). Utilização de dietas simplificadas à base de forragens sobre a digestibilidade e desempenho de coelhos da raça Nova Zelândia branco. Revista Brasileira de Cunicultura, 3 (1), 1-12.

Pereira, A. S., Shitsuka, D. M., Parreira, F. J., Shitsuka, R. (2018). Metodologia da pesquisa científica. UFSM.

Safwat, A. M., Franco, L. S., Ricalde, R. S., Nieves, D. (2014). Effect of dietary inclusion of Leucaena leucocephala or Moringa oleifera leaf meal on performance of growing rabbits. Tropical Animal Health and Production. 46 (6), 1193-1198. 10.1007/s11250-014-0626-1. 
Research, Society and Development, v. 10, n. 13, e492101321473, 2021 (CC BY 4.0) | ISSN 2525-3409 | DOI: http://dx.doi.org/10.33448/rsd-v10i13.21473

Santos Garcia, S., Almeida, M., Closson, M., Guedes, C. M., Barros, A., Ferreira, L. M., Trindade, H., \& Pinheiro, V. (2021). Effect of total replacement of the soya bean meal by lupine seeds (L. albus and L. luteus) on performance and digestion characteristics of growing rabbits. Animal Feed Science and Technology. 278(8), 1-9. https://doi.org/10.1016/j.anifeedsci.2021.114996.

Santos R. J., Gutiérrez Ruiz, E., Novelo Ucan, W., Martinez, R. P., \& Correa, J. S. (2017). Effect of feed restriction on intake of Moringa oleifera and Leucaena leucocephala and growth performance of rabbits. Tropical Animal Health and Production, 49 (8), 1685-1688. 10.1007/s11250-017-1377-6.

Strychalski, J., Juskiewicz, J., Gugolek, A., Wyczling, P., Daszkiewciz, T., \& Zwolinski, C. (2014). Usability of rapeseed cake and wheat-dried distillers' grains with solubles in the feeding of growing Californian rabbits. Archives of Animal Nutrition, 68 (3), 227-244. 10.1080/1745039X.2014.921482.

Turchi, B., Mancini, S., Pastorelli, R., Viti, C., Tronconi, L., Bertelloni, F., Felicioli, A., Cerri, D., Fratini, F., \& Paci, G. (2019). Dietary supplementation of chestnut and quebracho tannins mix: Effect on caecal microbial communities and live performance of growing rabbits. Research in Veterinary Science, 124 (6), 129-136. https://doi.org/10.1016/j.rvsc.2019.03.012.

Viana, F. J. C., Araújo, D. C., Sousa, A. R., Reis, A.L., Camargo, P. H. G., Santana, T. S. B., \& Biogiotti, D. (2018). Alimentos alternativos para a cunicultura do semiárido. Revista Brasileira de Cunicultura. 14(1), 1-13, 2018.

Wang, C., Zhu, Y., Li, F., \& Huang, L. (2017). The Effect of Lactobacillus isolates on growth performance, immune response, intestinal bacterial community composition of growing Rex Rabbits. Journal of Animal Physiology and Animal Nutrition, 101(5), 1-13. 10.1111/jpn.12629.

Yang, Y., Shen, Y., Pan, Y., Xia, P., Zhang, D., He, Z., Yang, J., Li, H., \& Lu, J. (2018). Effects of dietary sorghum dried distiller's grains with solubles on growth performance, diet nutrient digestibility, carcass characteristics and immunity in growing rabbits. Journal of Animal Physiology and Animal Nutrition, 103 (1), 1-7. 10.1111/jpn.13008. 